\title{
Natural history of aortic root aneurysms in Marfan syndrome
}

\author{
Ayman Saeyeldin ${ }^{1}$, Mohammad A. Zafar ${ }^{1}$, Camilo A. Velasquez ${ }^{1}$, Kevan Ip ${ }^{1}$, Anton Gryaznov ${ }^{1,2}$, Adam J. \\ Brownstein $^{1}$, Yupeng $\mathrm{Li}^{3}$, John A. Rizzo ${ }^{4}$, Young Erben ${ }^{5}$, Bulat A. Ziganshin ${ }^{1,6}$, John A. Elefteriades ${ }^{1}$ \\ ${ }^{1}$ Aortic Institute at Yale-New Haven Hospital, Yale University School of Medicine, New Haven, CT, USA; ${ }^{2}$ Department of Pharmacology, Kazan \\ State Medical University, Kazan, Russia; ${ }^{3}$ Department of Political Sciences and Economics, Rowan University, Glassboro, NJ, USA; ${ }^{4}$ Department of \\ Economics and Preventive Medicine, Stony Brook University, Stony Brook, NY, USA; ${ }^{5}$ Department of Vascular Surgery, Yale University School of \\ Medicine, New Haven, CT, USA; ${ }^{6}$ Department of Surgical Diseases \#2, Kazan State Medical University, Kazan, Russia \\ Correspondence to: John A. Elefteriades, MD. Aortic Institute at Yale-New Haven, Yale University School of Medicine, Clinic Building CB 317,789 \\ Howard Avenue, New Haven, CT 06519, USA. Email: john.elefteriades@yale.edu.
}

\begin{abstract}
Background: Cardiovascular complications account for a significant proportion of the shortened lifespan of Marfan syndrome (MFS) patients, with aortic dissection being the most dreadful complication. The aortic root dilates initially in MFS patients, and given its important hemodynamic role, this can lead to aortic regurgitation and poses a substantial risk of aortic dissection. This study seeks to evaluate the natural history of aortic root aneurysms in MFS patients, with a focus on growth rates and correlation of root diameter with the risk of developing aortic complications.

Methods: Seventy-eight patients confirmed to have MFS and aortic root dilatation were retrospectively reviewed, and their aortic root diameters serially analyzed. Annual growth rate estimates and yearly rates of adverse events were computed and correlated with aortic diameter.

Results: The mean annual growth rate of the aortic root was estimated to be $0.26 \pm 0.05 \mathrm{~cm} /$ year (range 0.13 to $0.35 \mathrm{~cm}$ ). Larger aneurysms grew faster, reaching up to $0.46 \mathrm{~cm} / \mathrm{year}$ for aneurysms $>6 \mathrm{~cm}$. Mean age at onset of aortic dissection was $36 \pm 4$ years. Annual rates of adverse events (rupture, dissection and death) were obtained using a logistic regression model at sizes 3.5, 4, 4.5, 5, 5.5 and $6 \mathrm{~cm}$. A sharp increase of $23 \%$ in the probability of the risk of complications at diameters 5.5 to $6 \mathrm{~cm}$ was recognized.

Conclusions: Aortic root aneurysms in MFS patients tend to have a faster expansion rate compared to non-MFS individuals, with aortic root diameter having a significant impact on the yearly risk of developing aortic complications.
\end{abstract}

Keywords: Thoracic aortic aneurysm; Marfan syndrome; natural history; aortic complications; aortic root

Submitted Nov 09, 2017. Accepted for publication Nov 21, 2017.

doi: $10.21037 /$ acs.2017.11.10

View this article at: http://dx.doi.org/10.21037/acs.2017.11.10

\section{Introduction}

In 1896, the French pediatrician Antoine Marfan described the first patient with arachnodactyly. In the interim, additional phenotypic features have been described to fully elucidate Marfan syndrome (MFS), including the notorious association with aortic root aneurysms and aortic dissection.

MFS (MIM \#154700) is the most common connective tissue disorder, with an incidence of 1 in 10,000. (1) MFS is autosomal dominantly inherited and associated with $F B N-1$ gene mutations located on chromosome $15 q 21$ (2).
The alteration in the microfibril protein structure results in the classic stigmata of ocular, skeletal, and cardiovascular abnormalities, such as mitral valve prolapse (MVP) and aortic root dilatation $(3,4)$. Among these, cardiovascular complications account for a significant proportion of the shortened lifespan of MFS patients $(5,6)$.

Approximately $60-80 \%$ of adult patients with MFS ultimately develop aortic root dilatation (7), with the prevalence being higher in males than in females (8). The aortic root comprises the aortic valve leaflets, the sinuses, the commissures, and the inter-leaflet triangles. The sinuses 
play a very important role in maintaining valve function by providing a space behind the aortic leaflets so that they do not occlude the coronary arteries orifices (9). This space also favors the development of eddy currents behind the leaflets, holding them away from the aortic wall and allowing them to close properly at the end of systole $(9,10)$. Dilatation of the aortic root alters the normal physiology of the aortic valve, leading to aortic regurgitation. This is a result of annular dilation and outward displacement of the valve commissures, leading to an inability of the leaflet edges to coapt in diastole (11). Many patients succumb to the dreadful complication of aortic dissection, which generally begins just above the coronary ostia and can extend to involve the entire length of the aorta (a type-A dissection according to the Stanford classification, or type I dissection per the DeBakey classification).

Previous work by our group has addressed the natural history of thoracic aortic aneurysms (TAAs) (12-17); herein, the aim of our current study is to assess the natural course of the aortic root specifically in MFS patients. We feel that the focus on the MFS aortic root may produce information of clinical value, as the root is the source of lethal complications in these patients. The focus of this study is (I) to determine aortic root growth rates; and (II) to correlate complications (namely rupture, dissection, and death) with aortic root size in this patient cohort.

\section{Methods}

This study was approved by the Human Investigation Committee of the Yale University School of Medicine.

\section{Patient population}

Our database at the Aortic Institute at Yale-New Haven Hospital (YNHH) includes a total of 3,349 TAA patients' records. Among these, a small subset of 78 patients confirmed to have MFS (by Ghent criteria and/or genetic confirmation) with documented dilatation of the aortic root were retrospectively reviewed. A total of 191 serial aortic root aneurysm size measurements were retrieved and verified over a median follow up of $150 \pm 85$ months.

Anthropologic, radiologic and clinical data were retrospectively obtained from the hospital's electronic medical records system and from the paper charts. Forty-six (59\%) of the patients in this subset were males and 32 (41\%) were females. Mean age at presentation was $48.2 \pm 14$.6 years (range 22 to 83 years).
Included patients were confirmed to have MFS by meeting the 2010 revised Ghent criteria (18), which puts greater weight on aortic pathology, systemic features, and ectopia lentis as the cardinal features of MFS, and on genetic testing of the $F B N-1$ mutation when available. The aortic criterion was defined as having a $Z$ score $\geq 2$ or aortic dissection. Systemic score included multiple features such as thumb sign, pectus excavatum or scoliosis. A patient was considered to have MFS if they met the aortic criterion plus either ectopia lentis, $F B N-1$ mutation or a systemic score $\geq 7$ (18).

Genetic testing was conducted in 22 patients (28\%), confirming the FBN-1 gene mutation. Among these, one patient also showed a likely pathogenic variant of the TGFBR2 gene, associated with Loeys-Dietz syndrome (LDS), and one patient showed a likely pathogenic variant of the COL5A1 gene, associated with the classic type Ehlers-Danlos syndrome (EDS). These two patients were included in this study since they met the aortic and systemic score criteria of MFS, expressed the FBN-1 mutation, and had none of the discriminating features of EDS or LDS described in the Ghent nosology (18). Such findings of nonMFS mutations in patients diagnosed clinically with MFS are being increasingly recognized in the present era of whole exome sequencing (WES) for aortic disease patients (19).

All patients had aneurysmal dilatation of the aortic root, with at least one verified aortic measurement prior to the development of an endpoint (rupture, dissection and death). The aortic root was defined as the portion of the left ventricular outflow tract which supports the leaflets of the aortic valve, delineated by the sinotubular junction superiorly and the bases of the valve leaflets inferiorly, and comprising the sinuses, the aortic valve leaflets, the commissures, and the interleaflet triangles (9). The aortic root was considered aneurysmal once it attained a maximal diameter of $3.5 \mathrm{~cm}$ or greater.

Primary endpoints for this study at which we stopped tracking the natural history were surgical repair of the aortic root, with or without aortic valve replacement, development of acute type-A aortic dissection involving the root, development of aortic rupture, or death.

Survival follow-up of the patients was performed according to the Yale Aortic Institute methods described previously (20).

Exclusion criteria for this study were associated congenital aortic malformations (e.g., coarctation of aorta), history of traumatic aortic rupture, and patients who had no radiographic data prior to the development of an endpoint. Patients with chronic type-A aortic dissection were also excluded since dissection was considered as an endpoint, 
hence ending the natural history. Patients' characteristics are listed in Table 1.

Nine patients developed aortic dissection distal to the graft after their aortic root repair, and four patients developed type-B aortic dissection without involvement of the ascending aorta. These patients were not included in the statistical analysis.

Bicuspid aortic valves were confirmed by transthoracic echocardiography (TTE), transesophageal echocardiography (TEE) or by direct visualization during surgery in patients who underwent repair. Bovine aortic arch was defined as a common origin of the brachiocephalic and left common carotid artery. A bovine configuration was confirmed by computed tomography (CT) or by magnetic resonance imaging (MRI).

Family history was considered positive if a relative of the patient had a TAA or aortic dissection confirmed on an imaging study (CT, MRI, TTE or TEE), intraoperatively or on autopsy, including affected relatives alive or expired.

\section{Imaging}

Radiographic imaging data were accrued using TTE or TEE in 55 patients, CT in 16 patients, and MRI in 7 patients. For serial measurement follow up, the same imaging modality was utilized each time at the identical level and plane. Official reports from the Department of Radiology at $\mathrm{YNHH}$ were reviewed, and radiographic measurements were doubly confirmed by the senior author (John A. Elefteriades) or by another senior team member (Mohammad A. Zafar). Measurements from echocardiography were preferred (due to its efficacy in visualizing the root) and measurements taken using the leading-edge-to-leading-edge technique, in diastole (21).

Aortic rupture and acute flap-type aortic dissection were confirmed by imaging (CT or MRI), autopsy or operation.

\section{Statistical methods}

Statistical analysis was performed using R 3.1.0 (R Foundation for Statistical Computing, Vienna, Austria) (22).

Serial measurements of 70 patients were used to obtain growth rate estimates. Patients who expired were excluded from the growth rate analysis due to the difficulty of verifying accurate measurements before death. The clinically observed growth rate was arithmetically calculated and was also estimated using the instrumental variables approach described previously by our group (23), in which the change in aneurysm growth followed an exponential path, and the natural logarithm of the subsequent measured size to the previous measured size was related to the time interval between the two tests.

In order to generate an estimate of growth rate for larger aneurysms, we used the estimated regression coefficient from our model and multiplied this by an initial aneurysm size of $6 \mathrm{~cm}$. But in the actual data, no patient had an initial aneurysm size of $6 \mathrm{~cm}$, since such patients would typically be operated on rather than followed for further growth progression. To obtain the growth estimates for these patients, we used the coefficients of the estimated model but applied an initial aneurysm size that was larger than was observed in the data.

Since Marfan patients with an aneurysm size of $6 \mathrm{~cm}$ would very likely be operated on (if not sooner than reaching $6 \mathrm{~cm}$ ), there is simply no other feasible way to obtain a growth rate estimate for such patients.

A logistic regression model was utilized to calculate the probability of adverse events, specifically a composite of aortic rupture, aortic dissection, and death. The adverse events were correlated with the aneurysm diameter as the risk factor. The estimated effect of root aneurysm size on the risk of complications was depicted using a percentage point increase in the probability of complications against a reference point of sizes $<4 \mathrm{~cm}$.

\section{Results}

\section{Aneurysm characteristics \& growth rates}

The exponential model for growth rate estimation assumed that aneurysm growth rate increased with initial aortic diameter. The average aneurysm diameter at presentation for our sample was $4.54 \pm 1.3 \mathrm{~cm}$ (range 2.2 to $8.3 \mathrm{~cm}$ ). The annual growth rate was estimated to be $0.26 \pm 0.05 \mathrm{~cm} /$ year. The minimum growth rate (for all the patients within the data set) was $0.132 \mathrm{~cm}$ and the maximum growth rate was $0.355 \mathrm{~cm}$ (Table 2). Figure 1 depicts the aneurysm growth rates in a density curve graph, comparing the observed growth rate with the statistically estimated growth rate.

Estimated growth rates were found to be faster in larger aneurysms (Figure 2), reaching up to $0.46 \mathrm{~cm} /$ year for aneurysms $>6 \mathrm{~cm}$, compared to the mean annual growth rate of $0.26 \mathrm{~cm} /$ year for the average aneurysm size $(4.6 \mathrm{~cm})$.

\section{Endpoints}

Forty-eight patients (62\%) underwent surgical repair of 


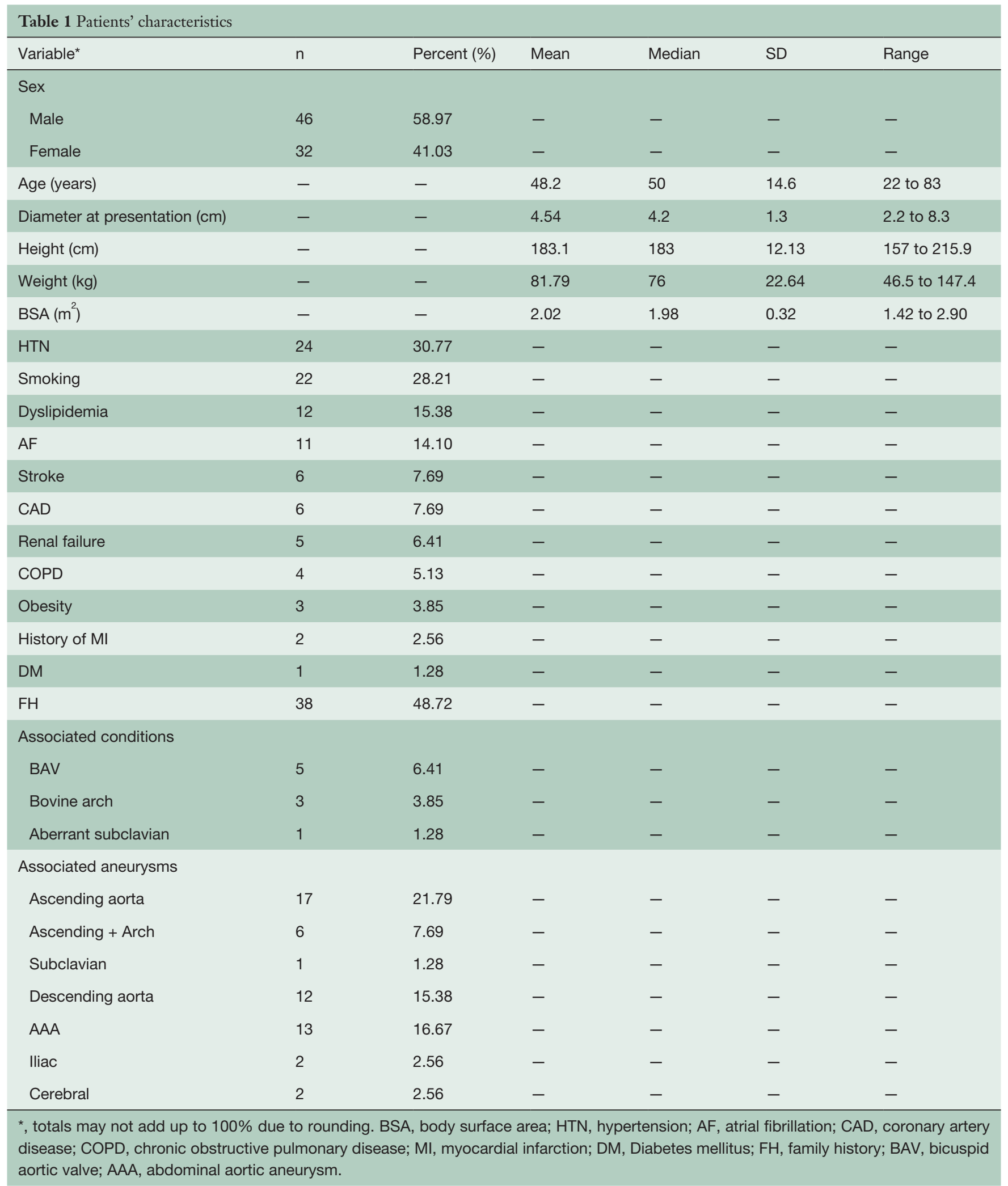




\begin{tabular}{lcccccccc} 
Table 2 Estimated and observed growth rates & & & & & & \\
\hline Statistic & $\mathrm{n}$ & Mean & $\begin{array}{l}\text { Standard } \\
\text { deviation }\end{array}$ & Minimum & Percentile 25\% Median & Percentile 75\% Maximum \\
\hline Estimated growth rate & 70 & 0.260 & 0.055 & 0.132 & 0.217 & 0.247 & 0.313 & 0.355 \\
Observed growth rate & 70 & 0.364 & 0.583 & 0.000 & 0.036 & 0.149 & 0.336 & 2.090 \\
\hline
\end{tabular}

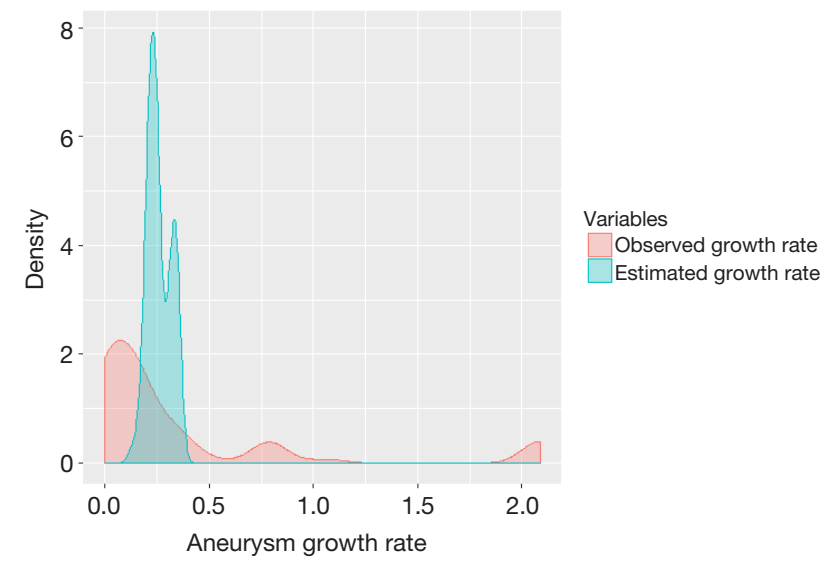

Figure 1 Growth distribution curve of the aortic root in Marfan syndrome patients. Observed growth rates are shown under the red curve, while the statistically estimated growth rates are shown under the blue curve. The mean aneurysm size from the data $(4.5 \mathrm{~cm})$ is different from the mean aneurysm size in the figure since the latter is obtained from the regression analysis.

their root aneurysms with coronary artery re-implantation into the graft.

The average age at the time of developing aortic dissection was $36 \pm 4$ years. The presenting event in six patients was type-A dissection involving the root, and one patient presented with aortic rupture, all of whom were managed surgically.

Eight patients (10\%) expired during the total follow-up period. Among these, two patients died of aortic dissection, one patient died of methicillin-resistant Staphylococcus aureus (MRSA) septicemia complicating a type-A dissection repair, one patient had a possibly aortic related death (chest pain), and four patients had non-aortic deaths.

\section{Complication rates}

The incidence of complications increased as the aortic size enlarged. The average yearly rates of adverse events for six

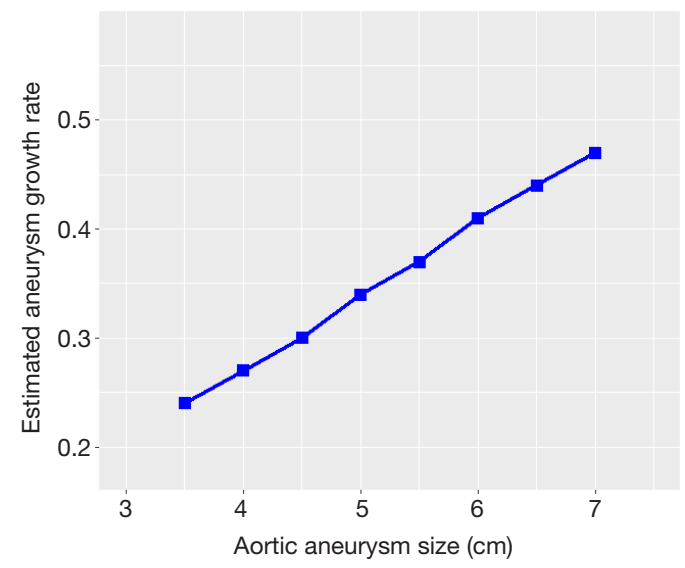

Figure 2 Estimated annual growth rate of the aneurysmal aortic root in Marfan syndrome patients. Larger aneurysms tend to grow faster.

categories of aortic root sizes $(3.5,4,4.5,5,5.5$ and $6 \mathrm{~cm})$ are depicted in Figure 3, showing a risk of less than $8 \%$ in diameters less than $4 \mathrm{~cm}$, compared to $18 \%$ in diameters equal or greater than $6 \mathrm{~cm}$.

The analysis of the estimated probability of risk of complications revealed a nonlinear trend (Figure 4), depicting a sharp increase at diameters 5.5 to $6 \mathrm{~cm}$, attaining a $25 \%$ increase in the probability of complications, in comparison to the reference group. In this graph, the increased risk in the $4-4.9 \mathrm{~cm}$ cohort was not statistically significant.

\section{Discussion}

Remarkable progress has been achieved in understanding the pathogenesis of MFS, monitoring clinical status, preventing complications, and improving the management of aortic dissection, leading to dramatically increased life expectancy in this disease.

Accurate information on aortic size and rate of growth is vital to the clinician in assessing the patient's risk 


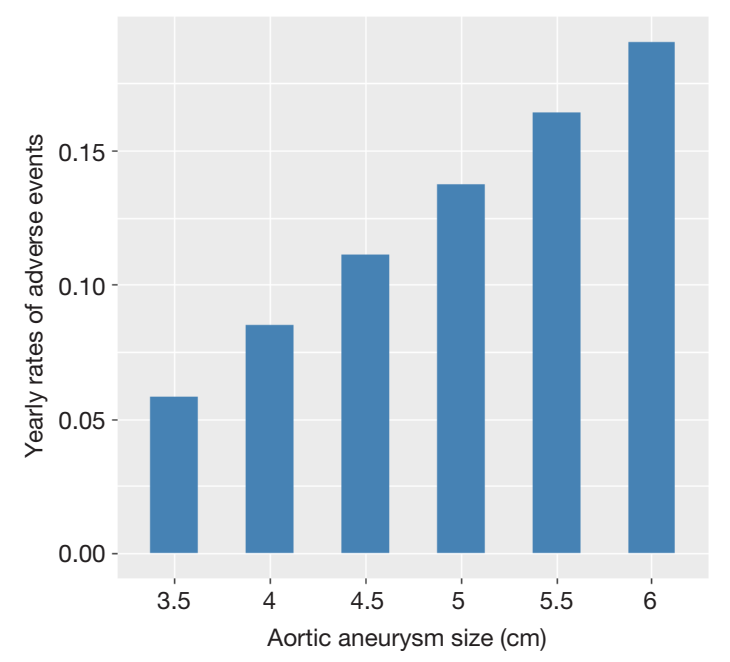

Figure 3 Annual rates of adverse events (rupture, dissection, and death) in Marfan syndrome patients.

and developing the treatment plan. Our study helps to determine the rate of growth in aortic root aneurysms specifically in MFS, with a mean growth of $0.26 \mathrm{~cm}$. This is significantly higher than the previously recognized $0.1 \mathrm{~cm} /$ year expansion rate in an overall thoracic aneurysm population (not exclusively MFS) individuals (25). Consistent with our previous findings in overall thoracic aortic aneurysm patients, aneurysm growth was found to increase with larger aneurysm sizes, reaching $0.4 \mathrm{~cm} /$ year at $6 \mathrm{~cm}$ and almost $0.46 \mathrm{~cm} /$ year at $7 \mathrm{~cm}$.

The instrumental variable approach (23) provides a more reliable estimate for aneurysm growth because it purges the effects of measurement error. An aneurysm size measurement may be inaccurate by as much as $0.5 \mathrm{~cm}$. The instrumental variables strategy is to correlate the change in measured aneurysm size (which includes measurement error) with a variable that is measured accurately-the time interval between the size measurements (e.g., we assume, as seems reasonable, that the dates when the tests were taken are accurate). Intuitively, measurement errorwhich is random noise-will be uncorrelated with the time interval between the measurements. Only the true growth rate should be correlated with the time interval variable. We prefer the instrumental variables approach because it mitigates problems of measurement errors inherent in estimates based on simple calculations of TAA growth rates, avoids the substantial fluctuations of observed growth rates that result from short-term serial follow-up periods, and yields much more robust statistical estimates with more

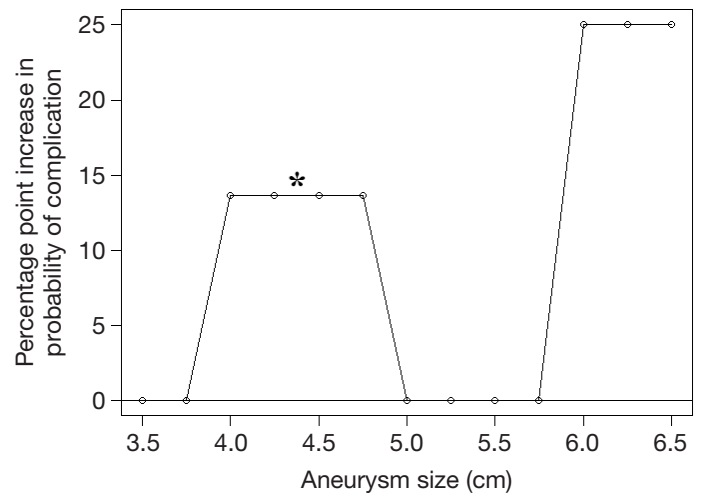

Figure 4 Estimated effect of aortic root aneurysm size on the risk of complications (rupture, dissection, and death) in Marfan syndrome patients. *, part of the hump between 4 and $4.75 \mathrm{~cm}$ is due to the skewed recognition of dissected patients in a small size range that would not normally be imaged. Our prior work on the "aortic size paradox" (24) indicates that because the tail of the bell curve thickens at smaller sizes, the relative rate of events is higher.

compact confidence intervals.

In regard to aortic dissection, the International Registry of Aortic Dissection (IRAD) addressed the frequency with which MFS is responsible for this complication $(26,27)$. The frequency varied with age. MFS was present in $50 \%$ of those under age 40 , compared to only $2 \%$ of older patients with aortic dissection. None of the patients presenting with aortic dissection over the age of 70 had MFS, which can be attributed to the shorter lifespan of MFS patients (5). Our results are consistent with these reports, with the average age of patients developing dissection in our cohort being 36 years, reflecting the early onset of complications in these patients.

Elective replacement of the aortic root before critical enlargement is tremendously preferable to emergency repair for marked dilatation or dissection. The importance of this approach was illustrated in a series of 675 patients with MFS from Johns Hopkins, in which the 30-day mortality for elective repair, urgent repair (within seven days of presentation), or emergency repair (within 24 hours of presentation) was $1.5 \%, 2.6 \%$, and $11.7 \%$, respectively. This highlights the importance of timely pre-emptive surgical intervention in these patients to improve their survival outcomes (28). The 2010 guidelines from the American College of Cardiology (ACC), American Heart Association (AHA) and the American Association for Thoracic Surgery (AATS) recommend elective operation 
for patients with MFS at an external diameter of $\geq 5 \mathrm{~cm}$ to avoid acute dissection or rupture (29).

Our results reveal that the yearly risk of developing complications increases with diameters more than $5 \mathrm{~cm}$, reaching $13 \%$, compared to $<8 \%$ in diameters less than $4 \mathrm{~cm}$. This is further highlighted in Figure 4, depicting a 'hinge point' between 5.5 and $6 \mathrm{~cm}$, where the probability of developing complications shoots up to $25 \%$. The discrepancy between these two charts (Figures 3 and 4) is explained by the different statistical approach used to plot them; the hinge point graph (Figure 4) uses aneurysm sizes as various categorical variables, while the bar chart (Figure 3) is based on a regression model where aneurysm size is a single continuous variable. These results from the present study support the recommendations for early prophylactic aortic root replacement in the MFS patient made in the society guidelines.

Despite much more detailed knowledge of the molecular, cellular, and tissue effects of the mutations in FBN1, there is a paucity of evidence regarding medical treatment, and targeted, effective therapy remains elusive. Some have argued that medical treatments are not really effective (30). The use of beta-blockers has been the standard for decades, but surgical extirpation of aortic aneurysms remains the most important factor in improving survival in MFS patients.

The present study provides valuable information regarding the natural history of the aortic root specifically in MFS patients. This data supports early pre-emptive extirpation of the dilated MFS root to prevent acute aortic dissection, rupture, and death-thus extending the lifespan of these patients.

\section{Limitations}

The study is limited by its single center, retrospective nature. Detailed survival analysis could not be conducted due to the small sample size, owing to the relative rarity of the condition and the strict inclusion criteria. Moreover, the study did not adjust for any effect of gender on developing adverse aortic events.

\section{Conclusions}

We have statistically analyzed behavior of the aortic root over time specifically in the MFS patient:

(I) Aortic root aneurysms in MFS patients tend to grow at $0.26 \mathrm{~cm} / \mathrm{year}$, significantly faster than in
non-MFS individuals, which may contribute to the increased risk of complications in these patients;

(II) The aortic root diameter remains a significant predictor of developing complications, with the risk increasing sharply at a 'hinge point' between 5.5 and $6 \mathrm{~cm}$;

(III) Preemptive surgical intervention before attaining these critical diameters is justified to prevent the dreadful complications of dissection, rupture, and death.

\section{Acknowledgements}

None.

\section{Footnote}

Conflicts of Interest: The authors have no conflicts of interest to declare.

\section{References}

1. Patel HJ, Deeb GM. Ascending and Arch Aorta. Circulation 2008;118:188-95.

2. Tsipouras P, Del Mastro R, Sarfarazi M, et al. Genetic Linkage of the Marfan Syndrome, Ectopia Lentis, and Congenital Contractural Arachnodactyly to the Fibrillin Genes on Chromosomes 15 and 5. N Engl J Med 1992;326:905-9.

3. Pyeritz RE, McKusick VA. The Marfan Syndrome: Diagnosis and Management. N Engl J Med 1979;300:772-7.

4. van Karnebeek CD, Naeff MS, Mulder BJ, et al. Natural history of cardiovascular manifestations in Marfan syndrome. Arch Dis Child 2001;84:129-37.

5. Murdoch JL, Walker BA, Halpern BL, et al. Life Expectancy and Causes of Death in the Marfan Syndrome. N Engl J Med 1972;286:804-8.

6. Adams JN, Trent RJ. Aortic complications of Marfan's syndrome. Lancet 1998;352:1722-3.

7. Roman MJ, Devereux RB, Kramer-Fox R, et al. Comparison of cardiovascular and skeletal features of primary mitral valve prolapse and Marfan syndrome. Am J Cardiol 1989;63:317-21.

8. Come PC, Fortuin NJ, White RI, et al. Echocardiographic assessment of cardiovascular abnormalities in the Marfan syndrome: Comparison with clinical findings and with roentgenographic estimation of aortic root size. Am J Med 
1983;74:465-74.

9. Underwood MJ, El Khoury G, Deronck D, et al. The aortic root: structure, function, and surgical reconstruction. Heart 2000;83:376-80.

10. Thubrikar M, Nolan SP, Bosher LP, et al. The cyclic changes and structure of the base of the aortic valve. Am Heart J 1980;99:217-24.

11. Bellhouse BJ, Bellhouse F, Abbott JA, et al. Mechanism of valvular incompetence in aortic sinus dilatation. Cardiovasc Res 1973;7:490-4.

12. Davies RR, Gallo A, Coady MA, et al. Novel measurement of relative aortic size predicts rupture of thoracic aortic aneurysms. Ann Thorac Surg 2006;81:169-77.

13. Coady MA, Rizzo JA, Hammond GL, et al. What is the appropriate size criterion for resection of thoracic aortic aneurysms? J Thorac Cardiovasc Surg 1997;113:476-91.

14. Davies RR, Goldstein LJ, Coady MA, et al. Yearly rupture or dissection rates for thoracic aortic aneurysms: simple prediction based on size. Ann Thorac Surg 2002;73:17-27; discussion 27-8.

15. Elefteriades JA. Natural history of thoracic aortic aneurysms: indications for surgery, and surgical versus nonsurgical risks. Ann Thorac Surg 2002;74:S1877-80.

16. Coady MA, Rizzo JA, Hammond GL, et al. Surgical intervention criteria for thoracic aortic aneurysms: a study of growth rates and complications. Ann Thorac Surg 1999;67:1922-6; discussion 1953-8.

17. Zafar MA, Li Y, Peterss S, et al. Height Alone (Rather than Body Surface Area) Suffices for Risk Estimation in Ascending Aortic Aneurysm. J Thorac Cardiovasc Surg 2017. (In Press).

18. Loeys BL, Dietz HC, Braverman AC, et al. The revised Ghent nosology for the Marfan syndrome. J Med Genet 2010;47:476-85.

19. Brownstein AJ, Ziganshin BA, Kuivaniemi H, et al. Genes Associated with Thoracic Aortic Aneurysm and Dissection: An Update and Clinical Implications. Aorta (Stamford) 2017;5:11-20.

20. Peterss S, Charilaou P, Ziganshin BA, et al. Assessment of survival in retrospective studies: The Social Security Death Index is not adequate for estimation. J Thorac Cardiovasc Surg 2017;153:899-901.

21. Muraru D, Maffessanti F, Kocabay G, et al. Ascending aorta diameters measured by echocardiography using both leading edge-to-leading edge and inner edge-to-inner edge conventions in healthy volunteers. Eur Heart J Cardiovasc Imaging 2014;15:415-22.
22. R Core Team. R: A Language and Environment for Statistical Computing. 3.1.0 ed. Vienna, Austria, 2014.

23. Rizzo JA, Coady MA, Elefteriades JA. Procedures for Estimating Growth Rates in Thoracic Aortic Aneurysms. J Clin Epidemiol 1998;51:747-54.

24. Paruchuri V, Salhab KF, Kuzmik G, et al. Aortic Size Distribution in the General Population: Explaining the Size Paradox in Aortic Dissection. Cardiology 2015;131:265-72.

25. Elefteriades JA, Farkas EA. Thoracic Aortic Aneurysm: Clinically Pertinent Controversies and Uncertainties. J Am Coll Cardiol 2010;55:841-57.

26. Januzzi JL, Isselbacher EM, Fattori R, et al. Characterizing the young patient with aortic dissection: results from the International Registry of Aortic Dissection (IRAD). J Am Coll Cardiol 2004;43:665-9.

27. Mehta RH, O'Gara PT, Bossone E, et al. Acute type A aortic dissection in the elderly: clinical characteristics, management, and outcomes in the current era. J Am Coll Cardiol 2002;40:685-92.

28. Gott VL, Greene PS, Alejo DE, et al. Replacement of the Aortic Root in Patients with Marfan's Syndrome. N Engl J Med 1999;340:1307-13.

29. Hiratzka LF, Bakris GL, Beckman JA, et al. 2010 ACCF/AHA/AATS/ACR/ASA/SCA/SCAI/SIR/STS/ SVM guidelines for the diagnosis and management of patients with Thoracic Aortic Disease: a report of the American College of Cardiology Foundation/American Heart Association Task Force on Practice Guidelines, American Association for Thoracic Surgery, American College of Radiology, American Stroke Association, Society of Cardiovascular Anesthesiologists, Society for Cardiovascular Angiography and Interventions, Society of Interventional Radiology, Society of Thoracic Surgeons, and Society for Vascular Medicine. Circulation 2010;121:e266-369.

30. Ziganshin BA, Mukherjee SK, Elefteriades JA. Atenolol versus Losartan in Marfan's Syndrome. N Engl J Med 2015;372:977-8.

Cite this article as: Saeyeldin A, Zafar MA, Velasquez CA, Ip K, Gryaznov A, Brownstein AJ, Li Y, Rizzo JA, Erben Y, Ziganshin BA, Elefteriades JA. Natural history of aortic root aneurysms in Marfan syndrome. Ann Cardiothorac Surg 2017;6(6):625-632. doi: 10.21037/acs.2017.11.10 